\title{
Generalized Powers of Graphs and Their Algorithmic Use
}

\author{
Andreas Brandstädt ${ }^{1}$, Feodor F. Dragan ${ }^{2}$, Yang Xiang ${ }^{2}$, and Chenyu Yan ${ }^{2}$ \\ 1 Universität Rostock, FB Informatik, Albert-Einstein-Str. 21, \\ D-18051 Rostock, Germany \\ ab@informatik.uni-rostock.de \\ 2 Department of Computer Science, Kent State University, Kent, OH 44242, USA \\ dragan, yxiang, cyan@cs.kent.edu
}

\begin{abstract}
Motivated by the frequency assignment problem in heterogeneous multihop radio networks, where different radio stations may have different transmission ranges, we introduce two new types of coloring of graphs, which generalize the well-known Distance-k-Coloring. Let $G=(V, E)$ be a graph modeling a radio network, and assume that each vertex $v$ of $G$ has its own transmission radius $r(v)$, a nonnegative integer. We define $r$-coloring $\left(r^{+}\right.$-coloring) of $G$ as an assignment $\Phi: V \mapsto\{0,1,2, \ldots\}$ of colors to vertices such that $\Phi(u)=\Phi(v)$ implies $d_{G}(u, v)>r(v)+r(u)\left(d_{G}(u, v)>r(v)+r(u)+1\right.$, respectively). The $r$-Coloring problem (the $r^{+}$-Coloring problem) asks for a given graph $G$ and a radius-function $r: V \mapsto N \cup\{0\}$, to find an $r$-coloring (an $r^{+}$coloring, respectively) of $G$ with minimum number of colors. Using a new notion of generalized powers of graphs, we investigate the complexity of the $r$-Coloring and $r^{+}$-Coloring problems on several families of graphs.
\end{abstract}

\section{Introduction}

The Frequency Assignment Problem (FAP) in multihop radio networks is the problem of assigning frequencies to transmitters exploiting frequency reuse while keeping signal interference to acceptable levels. The FAP is usually modeled by variations of the graph coloring problem. The $L\left(\delta_{1}, \delta_{2}, \ldots, \delta_{k}\right)$-coloring of a graph $G=(V, E)$, where $\delta_{i} \mathrm{~s}$ are positive integers, is an assignment function $\Phi: V \mapsto N \cup\{0\}$ such that $|\Phi(u)-\Phi(v)| \geq \delta_{i}$ when the distance between $u$ and $v$ in $G$ is equal to $i(i \in\{1,2, \ldots, k\})$. The aim is to minimize the range of the frequencies used, i.e., we search for the minimum $\lambda$ such that $G$ admits a $L\left(\delta_{1}, \delta_{2}, \ldots, \delta_{k}\right)$-coloring with frequencies between 0 and $\lambda$. Let us denote that minimum by $\lambda_{\delta_{1}, \delta_{2}, \ldots, \delta_{k}}(G)$. Unfortunately, already a restricted version of this problem, the $L(2,1)$-coloring problem (called also the Radiocoloring problem), is NP-complete even for planar graphs, bipartite graphs, chordal graphs and split graphs [5, 21], the classes of graphs where the ordinary graph coloring problem is easily (polynomial time) solvable. Polynomial time algorithms for optimal $L(2,1)$-coloring are known only for trees [11,24], cographs [11, $k$-almost trees [19] and for very regular graphs such as triangular grids, rectangular grids and hexagonal grids (see [4,10] and papers cited therein).

L. Arge and R. Freivalds (Eds.): SWAT 2006, LNCS 4059, pp. 423-434 2006.

(C) Springer-Verlag Berlin Heidelberg 2006 
Another variation of FAP considers $k$-powers, $G^{k}(k=1,2,3, \ldots)$, of a given graph $G$. The $k$ th power $G^{k}$ of $G=(V, E)$ has the same vertex set $V$ but two vertices $v$ and $u$ are adjacent in $G^{k}$ if and only if their distance in $G$ is at most $k$. The problem is to color the $k$ th power of $G$ with minimum number of colors, denoted by $\chi\left(G^{k}\right)$. This problem is extensively studied in literature and often called Distance- $k$-Coloring. Again, the problem is NP-complete even for chordal graphs [1] and for planar graphs and $k=2$ [31. Moreover, it is computationally hard to approximately color the even powers of $n$-vertex chordal graphs within an $n^{\frac{1}{2}-\epsilon}$ factor, for any $\epsilon>0$ 1. Exact polynomial time algorithms are known only for some special graph classes: for graphs with bounded tree-width [24, for graphs with bounded clique-width [33, and for interval, strongly chordal, doubly chordal, trapezoid, $d$-trapezoid, and cocomparability graphs as those families of graphs are closed under taking powers $[7,14,15,16,20,32$ and the ordinary coloring problem on them is polynomial time solvable 23, 26, 30. Approximation algorithms for coloring powers of chordal graphs and squares of planar graphs are presented in [2, 28.

The $L\left(\delta_{1}, \delta_{2}, \ldots, \delta_{k}\right)$-coloring problem and the Distance- $k$-Coloring problem are related. Clearly, $\chi\left(G^{k}\right)-1=\lambda_{\underbrace{1,1, \ldots, 1}_{k}}^{1, G)} \leq \lambda_{\delta_{1}, \delta_{2}, \ldots, \delta_{k}}(G)$ and, since from a valid coloring of $G^{k}$ we can always get a valid $L\left(\delta_{1}, \delta_{2}, \ldots, \delta_{k}\right)$-coloring of $G$ by multiplying by $t:=\max _{1 \leq i \leq k}\left\{\delta_{i}\right\}$ the assigned color of each vertex, we have also $\lambda_{\delta_{1}, \delta_{2}, \ldots, \delta_{k}}(G) \leq t\left(\chi\left(G^{k}\right)-1\right)$. Hence, an algorithm solving the Distance- $k$ Coloring problem for a class of graphs also provides a $\left(\max _{1 \leq i \leq k}\left\{\delta_{i}\right\}\right)$-approximation for the $L\left(\delta_{1}, \delta_{2}, \ldots, \delta_{k}\right)$-coloring problem. So, it is natural to investigate graph classes for which powers $G^{k}$ are easy to color.

In this paper, we extend the second variant of the Frequency Assignment Problem to the so-called heterogeneous multihop radio networks where different radio stations may have different transmission ranges. In this model, two radio stations $x$ and $y$ must not receive the same frequency if there is a third radio station $z$ which is within the transmission ranges of both $x$ and $y$ (to avoid collisions at $z$ ). In a more restricted model, we may forbid even two radio stations to have the same frequency if their transmission areas are very close. More formally, let $G=(V, E)$ be a graph modeling a radio network, and assume that each vertex $v$ of $G$ has its own transmission radius $r(v)$, a non-negative integer. We define $r$-coloring of $G$ as an assignment $\Phi: V \mapsto\{0,1,2, \ldots\}$ of colors to vertices such that $\Phi(u)=\Phi(v)$ implies $d_{G}(u, v)>r(v)+r(u)$, and $r^{+}$-coloring of $G$ as an assignment $\Phi: V \mapsto\{0,1,2, \ldots\}$ of colors to vertices such that $\Phi(u)=\Phi(v)$ implies $d_{G}(u, v)>r(v)+r(u)+1$. Here, $d_{G}(u, v)$ is the shortest path distance between $u$ and $v$ in $G$. The $r$-Coloring problem (the $r^{+}$-Coloring problem) asks for a given graph $G$ and a radius-function $r: V \mapsto N \cup\{0\}$, to find an $r$-coloring (an $r^{+}$-coloring, respectively) of $G$ with minimum number of colors. Clearly, if $r(v)=l$ ( $l$ is a fixed integer) for each $v \in V$, then $r$-coloring is just an ordinary coloring of $G^{2 l}$ and $r^{+}$-coloring is just an ordinary coloring of $G^{2 l+1}$. Hence, the $r$-Coloring and $r^{+}$-Coloring problems generalize the Distancek-Coloring. 


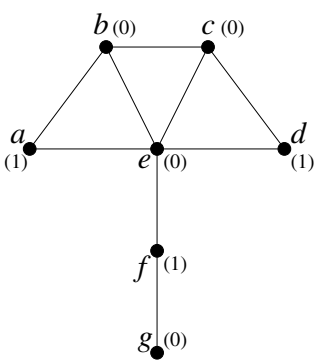

(a)

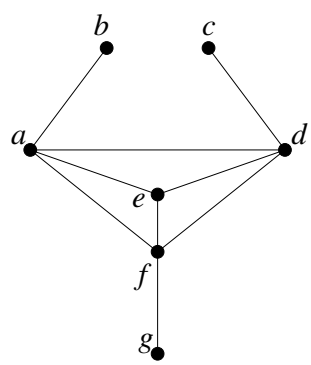

(b)

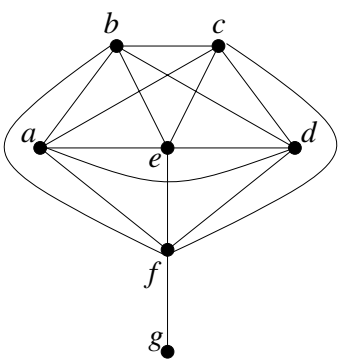

(c)

Fig. 1. A graph $G$ with a radius-function $r: V \mapsto N \cup\{0\}$ (a), and the corresponding graphs $L(\mathcal{D}(G, r))$ (b) and $\Gamma(\mathcal{D}(G, r))(\mathrm{c})$.

For a graph $G=(V, E)$ with a radius-function $r: V \mapsto N \cup\{0\}$, we can define two new graphs $\Gamma(\mathcal{D}(G, r))$ and $L(\mathcal{D}(G, r)$ ) (and call them generalized powers of $G)$ as follows. Both $\Gamma(\mathcal{D}(G, r))$ and $L(\mathcal{D}(G, r))$ have the same vertex set $V$ as $G$ has, and vertices $u, v \in V$ form an edge in $\Gamma(\mathcal{D}(G, r)$ ) (in $L(\mathcal{D}(G, r))$ ) if and only if $d_{G}(u, v) \leq r(v)+r(u)+1\left(d_{G}(u, v) \leq r(v)+r(u)\right.$, respectively). Figure 1 shows a graph $G$ with a radius-function $r: V \mapsto N \cup\{0\}$, and the corresponding graphs $\Gamma(\mathcal{D}(G, r))$ and $L(\mathcal{D}(G, r))$. It is easy to see that an $r$-coloring of $G$ is nothing else than an ordinary coloring of $L(\mathcal{D}(G, r))$ and an $r^{+}$-coloring of $G$ is nothing else than an ordinary coloring of $\Gamma(\mathcal{D}(G, r))$.

In this paper, we investigate the $r$-Coloring and $r^{+}$-Coloring problems on special graph classes. We are interested in determining large families of graphs $G$ for which the graphs $L(\mathcal{D}(G, r))$ and/or $\Gamma(\mathcal{D}(G, r))$ have enough structure to exploit algorithmically and to solve the $r$-Coloring and/or $r^{+}$-Coloring problems on $G$ efficiently. Among other results we show that

- if $G$ is a chordal (interval, circular-arc, cocomparability, weakly chordal) graph, then for any radius-function $r: V \mapsto N \cup\{0\}$, the graph $\Gamma(\mathcal{D}(G, r))$ is chordal (resp., interval, circular-arc, cocomparability, weakly chordal);

- if $G$ is a chordal graph with chordal square $G^{2}$ (a so called power-chordal graph), then for any radius-function $r: V \mapsto N \cup\{0\}$, the graphs $\Gamma(\mathcal{D}(G, r))$ and $L(\mathcal{D}(G, r))$ are chordal;

- if $G$ is a weakly chordal graph with weakly chordal square $G^{2}$, then for any radius-function $r: V \mapsto N \cup\{0\}$, the graphs $\Gamma(\mathcal{D}(G, r))$ and $L(\mathcal{D}(G, r))$ are weakly chordal;

- if $G$ is a distance-hereditary graph, then for any radius-function $r: V \mapsto$ $N \cup\{0\}$, the graph $\Gamma(\mathcal{D}(G, r))$ is weakly chordal and the graph $L(\mathcal{D}(G, r))$ is chordal;

- if $G$ is an AT-free graph, then for any radius-function $r: V \mapsto N$, the graphs $\Gamma(\mathcal{D}(G, r))$ and $L(\mathcal{D}(G, r))$ are cocomparability graphs (note that $r(v)=0$ is not allowed here);

- if $G$ is a cocomparability (interval, circular-arc) graph, then for any radiusfunction $r: V \mapsto N$, the graph $L(\mathcal{D}(G, r))$ is cocomparability (resp., interval, circular-arc). 
Since the (ordinary) coloring problem on chordal graphs, weakly chordal graphs, interval graphs and cocomparability graphs are polynomial time solvable (see [23, 26, 30]), we immediately obtain polynomial time solvability of the corresponding $r$-Coloring and/or $r^{+}$-Coloring problems on those graphs. Unfortunately, coloring of circular-arc graphs is an NP-complete problem [22]. However, one can use the circular-arc graph coloring approximation algorithm of [27] with a performance ratio of $3 / 2$ to get an approximate solution for the corresponding $r$-Coloring and/or $r^{+}$-Coloring problems on circular-arc graphs.

\section{Notations and Preliminaries}

Let $G=(V, E)$ be a finite, undirected, connected and simple (i.e. without loops and multiple edges) graph. For two vertices $x, y \in V$, the distance $d_{G}(x, y)$ is the length (i.e. number of edges) of a shortest path connecting $x$ and $y$. By $N_{G}(v)=\{u: u v \in E\}$ and $N_{G}[v]=N_{G}(v) \cup\{v\}$ we denote the open neighborhood and the closed neighborhood of $v$, respectively. If no confusion can arise we will omit the index $G$. Let $\mathcal{N}(G)=\{N[v]: v \in V\}$ be the family of closed neighborhoods of $G$. The disk centered at $v$ with radius $k$ is the set of all vertices having distance at most $k$ to $v: N^{k}[v]=\{u: u \in V$ and $d(u, v) \leq k\}$. Denote by $\mathcal{D}(G)=\left\{N^{r}[v]: v \in V, r\right.$ a non-negative integer $\}$ the family of all disks of $G$ and by $\mathcal{N}^{k}(G)=\left\{N^{k}[v]: v \in V\right\}$, where $k$ is a fixed non-negative integer, the family of all disks of radius $k$ of $G$. The $k$ th power of a graph $G=(V, E)$ is the graph $G^{k}=(V, U)$, where two vertices $x, y \in V$ are adjacent in $G^{k}$ if and only if $d_{G}(x, y) \leq k$.

For a graph $G$, consider a family $\mathcal{S}=\left\{S_{1}, \ldots, S_{l}\right\}$ of subsets of $V$, i.e., $S_{i} \subseteq V$, $i=1, \ldots, l$. The intersection graph $L(\mathcal{S})$ of $\mathcal{S}$ is defined as follows. The sets from $\mathcal{S}$ are the vertices of $L(\mathcal{S})$ and two vertices of $L(\mathcal{S})$ are joined by an edge if and only if the corresponding sets intersect. The visibility graph $\Gamma(\mathcal{S})$ of $\mathcal{S}$ is defined as follows. The sets from $\mathcal{S}$ are the vertices of $\Gamma(\mathcal{S})$ and two vertices of $\Gamma(\mathcal{S})$ are joined by an edge if and only if the corresponding sets are visible to each other. We say that sets $S_{i}$ and $S_{j}$ are visible to each other if $S_{i} \cap S_{j} \neq \emptyset$ or there is an edge of $G$ with one end in $S_{i}$ and the other end in $S_{j}$.

It is easy to see, from the definitions, that

- two disks $N^{p}[v]$ and $N^{q}[u]$ of $G$ are intersecting if and only if $d_{G}(u, v) \leq p+q$ and are visible to each other if and only if $d_{G}(u, v) \leq p+q+1$,

- $L\left(\mathcal{N}^{k}(G)\right)$ is isomorphic to $G^{2 k}(k \geq 1)$, i.e., $G^{2 k} \simeq L\left(\mathcal{N}^{k}(G)\right)$,

- $\Gamma\left(\mathcal{N}^{k}(G)\right)$ is isomorphic to $G^{2 k+1}(k \geq 0)$, i.e., $G^{2 k+1} \simeq \Gamma\left(\mathcal{N}^{k}(G)\right)$.

Definitions of graph classes considered are given in appropriate sections.

\section{3 c-Chordal Graphs}

In this section we consider the generalized powers of c-chordal graphs. A graph is called chordal if it has no induced cycles of size greater than 3 and is called $c$-chordal if it has no induced cycles of size greater than $c(c \geq 3)$. 
Let a maximal induced cycle of $G$ be an induced cycle of $G$ with maximum number of edges. Denote by $l(G)$ the number of edges of a maximal induced cycle of $G$. The parameter $l(G)$ of a graph $G$ is often called the chordality of $G$. Clearly, the chordal graphs are exactly the graphs of chordality 3 and $c$-chordal graphs are exactly the graphs of chordality $c$. In 7, an important lemma is proven which connects the chordality of $L(\mathcal{D}(G))$ with the chordality of $G^{2}$.

Lemma 1. [7] For any graph $G, l(L(\mathcal{D}(G)))=l\left(G^{2}\right)$.

One can prove a similar result for graphs $G$ and $\Gamma(\mathcal{D}(G))$ (proof is omitted).

Lemma 2. For any graph $G$ with $l(G) \geq 3, l(\Gamma(\mathcal{D}(G)))=l(G)$.

From Lemma 1 and Lemma 2 we conclude.

Theorem 1. For a graph $G$,

1) $\Gamma(\mathcal{D}(G))$ is c-chordal if and only if $G$ is c-chordal,

2) $L(\mathcal{D}(G))$ is c-chordal if and only if $G^{2}$ is c-chordal.

Let now $G=(V, E)$ be a graph and $r: V \mapsto N \cup\{0\}$ be a non-negative integervalued radius-function defined on $V$. For a graph $G$ with a radius-function $r$ : $V \mapsto N \cup\{0\}$, define a subfamily $\mathcal{D}(G, r)$ of the family of all disks $\mathcal{D}(G)$ of $G$ as follows: $\mathcal{D}(G, r)=\left\{N^{r(v)}[v]: v \in V\right\}$.

Clearly, graphs $L(\mathcal{D}(G, r))$ and $\Gamma(\mathcal{D}(G, r))$ are induced subgraphs of the graphs $L(\mathcal{D}(G))$ ) and $\Gamma(\mathcal{D}(G))$, respectively. Furthermore, the graph $L(\mathcal{D}(G, r))$ can be viewed (by identifying every disk with its center) as a graph on the vertex set $V$, where two vertices $u, v \in V$ are adjacent in $L(\mathcal{D}(G, r))$ if and only if $d_{G}(u, v) \leq r(u)+r(v)$. Similarly, the graph $\Gamma(\mathcal{D}(G, r))$ can be viewed as a graph on the vertex set $V$, where two vertices $u, v \in V$ are adjacent in $\Gamma(\mathcal{D}(G, r))$ if and only if $d_{G}(u, v) \leq r(u)+r(v)+1$. Thus, graphs $\Gamma(\mathcal{D}(G, r))$ and $L(\mathcal{D}(G, r))$ are generalizations of odd and even, respectively, powers of $G$.

Since induced subgraphs of $c$-chordal graphs are $c$-chordal, we can state the following corollaries from Lemma 1 and Lemma 2

Corollary 1. For any c-chordal graph $G$ and any radius-function $r: V \mapsto$ $N \cup\{0\}$ defined on the vertex set of $G$, the graph $\Gamma(\mathcal{D}(G, r))$ is c-chordal. In particular, odd powers $G^{2 k+1}(k=1,2, \ldots)$ of a c-chordal graph $G$ are $c$-chordal.

Corollary 2. Let $G$ be a graph having c-chordal square $G^{2}$. Then, for any radius-function $r: V \mapsto N \cup\{0\}$ defined on the vertex set of $G$, the graph $L(\mathcal{D}(G, r))$ is c-chordal. In particular, if the square $G^{2}$ of some graph $G$ is cchordal, then all even powers $G^{2 k}(k=1,2, \ldots)$ of $G$ are $c$-chordal.

Note that, Corollary 1 generalizes the known fact that odd powers of chordal graphs are chordal 1, 18, 29. Corollary 2 generalizes the known fact that even powers of square-chordal graphs are chordal [7,18. Here, a graph $G$ is squarechordal if its square $G^{2}$ is a chordal graph. Note also that the class of squarechordal graphs comprises such known families of graphs as trees, interval graphs, 
directed path graphs, strongly chordal graphs, doubly chordal graphs, distancehereditary graphs, dually chordal graphs, homogeneous graphs, homogeneously orderable graphs and others (see, for example, , 7, 8, 9, and papers cited therein). But, it still remains an open question to give a complete characterization of the whole family of square-chordal graphs. As it was shown in [29], squares of chordal graphs are not necessarily chordal; in fact, a square of a chordal graph can have arbitrarily large chordality 29 .

In [7, we defined power-chordal graphs as graphs $G$ all powers $G^{k}(k \geq 1)$ of which are chordal (or, equivalently, if both $G$ and $G^{2}$ are chordal). For this family of graphs we have.

Corollary 3. For a power-chordal graph $G$, graphs $\Gamma(\mathcal{D}(G, r)), L(\mathcal{D}(G, r))$ are chordal for any radius-function $r: V \mapsto N \cup\{0\}$ defined on the vertex set of $G$.

Notice that the class of power-chordal graphs comprises such known families of graphs as trees, interval graphs, directed path graphs, strongly chordal graphs and doubly chordal graphs [7].

\section{Weakly Chordal Graphs}

In this section we consider the generalized powers of weakly chordal graphs. In what follows, the complement of a graph $G$ is denoted by $\bar{G}, C_{k}$ is an induced cycle on $k$ vertices and $\overline{C_{k}}$ is the complement of $C_{k}$. A graph $G$ is weakly chordal if both $G$ and $\bar{G}$ are 4-chordal, i.e., $G$ has neither $C_{k}$ nor $\overline{C_{k}}, k>4$, as an induced subgraph.

Lemma 3. Let $G$ be a graph such that $\overline{L(\mathcal{N}(G))} \simeq \overline{G^{2}}$ is a 4-chordal graph and $L(\mathcal{D}(G))$ has no induced subgraphs isomorphic to $C_{5}$. Then, $\overline{L(\mathcal{D}(G))}$ is a 4-chordal graph, too.

Proof. Assume that the graph $\overline{L(\mathcal{D}(G))}$ is not 4-chordal. Then, there must exist an induced cycle $C_{k+1}$ in $\overline{L(\mathcal{D}(G))}$ such that $k+1>4$. In fact, $k+1$ is larger than 5 since $\overline{L(\mathcal{D}(G))}$ cannot have an induced subgraph isomorphic to $C_{5}$ (notice that an induced cycle on 5 vertices is self-complementary). We will assume that $k$ is minimal, i.e., any cycle of $\overline{L(\mathcal{D}(G))}$ of length $t(4<t \leq k)$ has a chord. Let $C_{k+1}$ be formed by disks $N^{r_{0}}\left[x_{0}\right], N^{r_{1}}\left[x_{1}\right], \ldots, N^{r_{k-1}}\left[x_{k-1}\right], N^{r_{k}}\left[x_{k}\right]$ of $G$, i.e., in $G$ we have $N^{r_{i}}\left[x_{i}\right] \cap N^{r_{j}}\left[x_{j}\right]=\emptyset$ if and only if $i=j \pm 1(\bmod (k+1))$. Among all such induced cycles $C_{k+1}$ of $\overline{L(\mathcal{D}(G))}$ we will choose one with minimum sum $\sigma:=r_{0}+r_{1}+\cdots+r_{k}$. Clearly, $r_{i}>0$ for each $i$. We will show that $r_{0}=r_{1}=\cdots=r_{k}=1$ holds.

Assume, without loss of generality, that $r_{0}>1$. Consider a neighbor $y$ of $x_{0}$ on a shortest path of $G$ from $x_{0}$ to $x_{2}$ and a neighbor $z$ of $x_{0}$ on a shortest path of $G$ from $x_{0}$ to $x_{3}$. Since $L(\mathcal{D}(G))$ has no induced subgraphs isomorphic to $C_{5}$, a cycle of $L(\mathcal{D}(G))$ formed by disks $N^{r_{0}-1}[y], N^{r_{0}-1}[z], N^{r_{2}}\left[x_{2}\right], N^{r_{3}}\left[x_{3}\right], N^{r_{k}}\left[x_{k}\right]$ cannot be induced. Therefore, as $N^{r_{0}}\left[x_{0}\right] \cap N^{r_{k}}\left[x_{k}\right]=\emptyset$, we must have $N^{r_{0}-1}[y] \cap$ $N^{r_{3}}\left[x_{3}\right] \neq \emptyset$ or $N^{r_{0}-1}[z] \cap N^{r_{2}}\left[x_{2}\right] \neq \emptyset$. Let, without loss of generality, $N^{r_{0}-1}[y] \cap$ $N^{r_{3}}\left[x_{3}\right] \neq \emptyset$. 
Thus, there is a neighbor $y$ of $x_{0}$ such that $N^{r_{0}-1}[y] \cap N^{r_{2}}\left[x_{2}\right] \neq \emptyset$ and $N^{r_{0}-1}[y] \cap N^{r_{3}}\left[x_{3}\right] \neq \emptyset$. Next we claim that $N^{r_{0}-1}[y] \cap N^{r_{i}}\left[x_{i}\right] \neq \emptyset$ for every $i=4, \ldots, k-1$. Let $i_{0}\left(3<i_{0}<k\right)$ be the minimal index such that $N^{r_{0}-1}[y] \cap$ $N^{r_{i_{0}}}\left[x_{i_{0}}\right]=\emptyset$. Then, it is easy to see that the disks $N^{r_{0}-1}[y], N^{r_{1}}\left[x_{1}\right], N^{r_{2}}\left[x_{2}\right]$, $N^{r_{3}}\left[x_{3}\right], \ldots, N^{r_{i_{0}}}\left[x_{i_{0}}\right]$ form an induced cycle in $\overline{L(\mathcal{D}(G))}$ of length $t:=i_{0}+1$ with $4<t \leq k$, and a contradiction with the choice of $k$ arises.

Now, disks $N^{r_{0}-1}[y]$ and $N^{r_{i}}\left[x_{i}\right]$ intersect if and only if $i \in\{2,3, \ldots, k$ $-2, k-1\}$, i.e., $i \neq 1, k$. But, then the disks $N^{r_{0}-1}[y], N^{r_{1}}\left[x_{1}\right], N^{r_{2}}\left[x_{2}\right], N^{r_{3}}$ $\left[x_{3}\right], \ldots, N^{r_{k-1}}\left[x_{k-1}\right], N^{r_{k}}\left[x_{k}\right]$ of $G$ induce a cycle in $\overline{L(\mathcal{D}(G))}$ of length $k+1$ and, since the sum of radiuses of these disks is $\sigma-1$, a contradiction with the minimality of $\sigma:=r_{0}+r_{1}+\cdots+r_{k}$ occurs.

Consequently, $r_{0}=r_{1}=\cdots=r_{k}=1$ must hold, implying that $C_{k+1}$ with $k+1>4$ is also an induced cycle of $\overline{L(\mathcal{N}(G))}$. The latter contradicts now with $\overline{L(\mathcal{N}(G))} \simeq \overline{G^{2}}$ being a 4-chordal graph. Obtained contradictions prove the lemma.

Combining Lemma 1 with Lemma 3 we obtain the following results. Notice that induced subgraphs of weakly chordal graphs are weakly chordal, too.

Theorem 2. For a graph $G, L(\mathcal{D}(G))$ is weakly chordal if and only if $G^{2}$ is weakly chordal.

Proof. By Lemma 1, $L(\mathcal{D}(G))$ is 4-chordal if and only if $G^{2}$ is so. Assuming now that $L(\mathcal{D}(G))$ is 4-chordal, by Lemma $3 \overline{L(\mathcal{D}(G))}$ is 4-chordal if and only if $\overline{G^{2}}$ is 4-chordal (notice that $G^{2}$ is an induced subgraph of $L(\mathcal{D}(G))$ ). Hence, $L(\mathcal{D}(G)$ ) is weakly chordal if and only if $G^{2}$ is weakly chordal.

Corollary 4. Let $G$ be a graph having weakly chordal square $G^{2}$. Then, for any radius-function $r: V \mapsto N \cup\{0\}$ defined on the vertex set of $G$, the graph $L(\mathcal{D}(G, r))$ is weakly chordal. In particular, if the square $G^{2}$ of some graph $G$ is weakly chordal, then all even powers $G^{2 k}(k=1,2, \ldots)$ of $G$ are weakly chordal.

Lemma 4. Let $G$ be the complement of a 4-chordal graph and $\Gamma(\mathcal{D}(G))$ has no induced subgraphs isomorphic to $C_{5}, C_{6}$. Then, $\overline{\Gamma(\mathcal{D}(G))}$ is a 4-chordal graph.

Proof of this lemma is omitted. Combining Lemma 2 with Lemma 4 we obtain the following results.

Theorem 3. For a graph $G, \Gamma(\mathcal{D}(G))$ is weakly chordal if and only if $G$ is weakly chordal.

Corollary 5. For any weakly chordal graph $G$ and any radius-function $r: V \mapsto$ $N \cup\{0\}$ defined on the vertex set of $G$, the graph $\Gamma(\mathcal{D}(G, r))$ is weakly chordal. In particular, odd powers $G^{2 k+1}(k=1,2, \ldots)$ of a weakly chordal graph $G$ are weakly chordal.

Recall (see, e.g., 9]) that a graph $G$ is distance-hereditary if each induced path of $G$ is shortest. It is known that any distance-hereditary graph $G$ is weakly chordal and its square $G^{2}$ is even chordal [3, 8. Hence the following result holds. 
Corollary 6. For any distance-hereditary graph $G$ and any radius-function $r$ : $V \mapsto N \cup\{0\}$ defined on the vertex set of $G$, the graph $\Gamma(\mathcal{D}(G, r))$ is weakly chordal and the graph $L(\mathcal{D}(G, r))$ is chordal. In particular [3, 8], odd powers $G^{2 k+1}(k=1,2, \ldots)$ of a distance-hereditary graph $G$ are weakly chordal, while its even powers $G^{2 k}(k=1,2, \ldots)$ are chordal.

\section{AT-Free Graphs and Cocomparability Graphs}

In this section we consider the generalized powers of AT-free graphs, cocomparability graphs and interval graphs.

In a graph $G$, an asteroidal triple is a triple of vertices such that between any two there is a path of $G$ that avoids the neighbourhood of the third. A graph $G$ is asteroidal triple-free (AT-free) if it has no asteroidal triples. It is shown in 12 that any AT-free graph $G=(V, E)$ admits a so-called strong 2-cocomparability ordering, i.e., an ordering $\sigma:=\left[v_{1}, v_{2}, \ldots, v_{n}\right]$ of vertices of $G$ such that for any three vertices $x, y, z$, if $x \prec y \prec z$ ( $x$ precedes $y$ and $y$ precedes $z$ in the ordering) and $d_{G}(x, z) \leq 2$ then $d_{G}(x, y)=1$ or $d_{G}(y, z) \leq 2$ must hold. Moreover, such an ordering of vertices of an AT-free graph $G=(V, E)$ can be found in time $O(|V|+|E|)[13]$.

Our next lemma shows that a strong 2-cocomparability ordering of an AT-free graph satisfies a useful distance property (proof is omitted).

Lemma 5. Let $G$ be an AT-free graph and $\sigma$ be a strong 2-cocomparability ordering of vertices of $G$. If $x \prec y \prec z$ and $d_{G}(y, z)>2$, then $d_{G}(x, y)+d_{G}(y, z) \leq$ $d_{G}(x, z)+3$.

It is well known (see, e.g., [16]) that a graph is cocomparability if and only if it admits a cocomparability ordering, i.e., an ordering $\sigma:=\left[v_{1}, v_{2}, \ldots, v_{n}\right]$ of its vertices such that if $x \prec y \prec z$ in $\sigma$ and $x z \in E(G)$ then $x y \in E(G)$ or $y z \in E(G)$ must hold. Lemma [5] is essential to proving the following result.

Theorem 4. Let $G=(V, E)$ be an AT-free graph and $r: V \mapsto N$ be a radiusfunction defined on $V$. Then, both $L(\mathcal{D}(G, r))$ and $\Gamma(\mathcal{D}(G, r))$ are cocomparability graphs.

Proof. Using Lemma 5, we will show that any strong 2-cocomparability ordering $\sigma$ of vertices of $G$ gives a cocomparability ordering for both $L(\mathcal{D}(G, r))$ and $\Gamma(\mathcal{D}(G, r))$. In what follows, we will identify a vertex $N^{r(v)}[v]$ of $L(\mathcal{D}(G, r))$ (and $\Gamma(\mathcal{D}(G, r))$ ) with $v$.

Assume, by way of contradiction, that there exist three vertices $x, y, z$ in $L(\mathcal{D}(G, r))$ such that $x \prec y \prec z$ in $\sigma, x z \in E(L(\mathcal{D}(G, r)))$ but neither $x y$ nor $y z$ is in $E(L(\mathcal{D}(G, r)))$. We know that two vertices $u, v \in V$ are adjacent in $L(\mathcal{D}(G, r))$ if and only if $d_{G}(u, v) \leq r(u)+r(v)$. Hence, we have $d(x, y) \geq r(x)+r(y)+1$, $d(y, z) \geq r(y)+r(z)+1$ and $d(x, z) \leq r(x)+r(z)$, i.e., $d(x, y)+d(y, z) \geq$ $d(x, z)+2 r(y)+2$. Since, by the theorem assumption, $r(v) \geq 1$ for any $v \in V$, we obtain $d(x, y)+d(y, z) \geq d(x, z)+4$, which is in a contradiction with Lemma 5 
(note that $d(y, z) \geq r(y)+r(z)+1 \geq 3>2$ ). Thus, a strong 2-cocomparability ordering $\sigma$ of vertices of $G$ must be a cocomparability ordering for $L(\mathcal{D}(G, r)$ ), i.e., $L(\mathcal{D}(G, r))$ is a cocomparability graph.

Assume now, by way of contradiction, that there exist three vertices $x, y, z$ in $\Gamma(\mathcal{D}(G, r))$ such that $x \prec y \prec z$ in $\sigma, x z \in E(\Gamma(\mathcal{D}(G, r)))$ but neither $x y$ nor $y z$ is in $E(\Gamma(\mathcal{D}(G, r)))$. We know that two vertices $u, v \in V$ are adjacent in $\Gamma(\mathcal{D}(G, r))$ if and only if $d_{G}(u, v) \leq r(u)+r(v)+1$. Hence, we have $d(x, y) \geq$ $r(x)+r(y)+2, d(y, z) \geq r(y)+r(z)+2$ and $d(x, z) \leq r(x)+r(z)+1$, i.e., $d(x, y)+d(y, z) \geq d(x, z)+2 r(y)+3$. Since, by the theorem assumption, $r(v) \geq 1$ for any $v \in V$, we obtain $d(x, y)+d(y, z) \geq d(x, z)+5$, which is in a contradiction with Lemma 5 (note that $d(y, z) \geq r(y)+r(z)+2 \geq 4>2$ ). Thus, a strong 2cocomparability ordering $\sigma$ of vertices of $G$ must be a cocomparability ordering for $\Gamma(\mathcal{D}(G, r))$, i.e., $\Gamma(\mathcal{D}(G, r))$ is a cocomparability graph, too.

Notice that Theorem 4 is not true if we allow $r(v)=0$ for some vertices $v$ of $G$. An induced cycle $C_{5}$ on five vertices is an AT-free graph, however $\Gamma\left(\mathcal{D}\left(C_{5}, r\right)\right)$, where $r(v)=0$ for each vertex $v$ of $C_{5}$, is not a cocomparability graph (since $\Gamma\left(\mathcal{D}\left(C_{5}, r\right)\right) \simeq C_{5}$ and $C_{5}$ is not a cocomparability graph). The graph $G$ shown in Figure 1 is an AT-free graph (even an interval graph), however the graph $L(\mathcal{D}(G, r))$ shown in that figure contains an asteroidal triple $b, c, g$.

As a corollary, we obtain the following result known from [12].

Corollary 7. [12] If $G$ is an AT-free graph, then $G^{k}$ is a cocomparability graph for any $k \geq 2$.

Recall (see, e.g., 9]) that any cocomparability graph is AT-free. Therefore, Theorem 4 holds for any cocomparability graph, too. However, for the class of cocomparability graphs a slightly stronger result can be proven. In [16], it was shown that if $G$ is a cocomparability graph and $\sigma$ is its cocomparability ordering, then $x \prec y \prec z$ implies $d_{G}(x, y)+d_{G}(y, z) \leq d_{G}(x, z)+2$. Using this stronger version of Lemma 5, similar to the proof of Theorem 4, one can prove the following.

Theorem 5. Let $G=(V, E)$ be a cocomparability graph. Then, for any radiusfunction $r: V \mapsto N, L(\mathcal{D}(G, r))$ is a cocomparability graph, and for any radiusfunction $r: V \mapsto N \cup\{0\}, \Gamma(\mathcal{D}(G, r))$ is a cocomparability graph.

Corollary 8. [16] All powers $G^{k}(k \geq 1)$ of a cocomparability graph $G$ are cocomparability, too.

Note that the class of cocomparability graphs contains such known families of graphs as interval graphs, permutation graphs, trapezoid graphs and $m$-trapezoid graphs. Hence, the graphs $L(\mathcal{D}(G, r))$ and $\Gamma(\mathcal{D}(G, r))$ for a graph $G$ from those families are cocomparability, too. For interval graphs the result can be further strengthened. An interval graph is the intersection graph of intervals of a line.

Theorem 6. Let $G=(V, E)$ be an interval graph. Then, for any radius-function $r: V \mapsto N, L(\mathcal{D}(G, r))$ is an interval graph, and for any radius-function $r: V \mapsto$ $N \cup\{0\}, \Gamma(\mathcal{D}(G, r))$ is an interval graph. 
Corollary 9. 14] All powers $G^{k}(k \geq 1)$ of an interval graph $G$ are interval.

Due to space limitation, we omit results on circular-arc graphs. In the full version of this paper, we show that for any circular-arc graph $G$ and any radius-function $r: V \mapsto N$, both graphs $L(\mathcal{D}(G, r))$ and $\Gamma(\mathcal{D}(G, r))$ are circular-arc.

\section{Algorithmic Use of the Generalized Powers of Graphs}

Based on the results obtained in the previous sections and known results on ordinary coloring, we deduce the following complexity results for the $r$-Coloring and $r^{+}$-Coloring Problems on the graph families considered in this paper (see Table 10.

Table 1. Complexity results for the $r$-Coloring and $r^{+}$-Coloring Problems on the graph families considered in this paper. ${ }^{(*)}$ marking means that $r(v)=0$ is not allowed. Here we define the power-4-chordal (the power-weakly-chordal) graphs as graphs $G$ for which both $G$ and $G^{2}$ (equivalently, all powers of $G$ ) are 4-chordal (respectively, weakly chordal) graphs. If for a graph $G$, only $G^{2}$ is 4 -chordal (is weakly chordal), then we say that $G$ is a square-4-chordal (respectively, a square-weakly-chordal) graph.

\begin{tabular}{|c|c|c|}
\hline $\begin{array}{c}\text { Graph } \\
\text { class }\end{array}$ & $\begin{array}{c}\text { Complexity of the } \\
\text { r-Coloring problem }\end{array}$ & $\begin{array}{c}\text { Complexity of the } \\
r^{+} \text {-Coloring problem }\end{array}$ \\
\hline \hline chordal & hard to approximate & $O(n m)$ \\
\hline 4-chordal & hard to approximate & $P$ \\
\hline weakly chordal & hard to approximate & hard to approximate \\
\hline square-chordal & $O(n m)$ & hard to approximate \\
\hline square-4-chordal & $P$ & hard to approximate \\
\hline square-weakly-chordal & $O\left(n^{3}\right)$ & $O(n m)$ \\
\hline power-chordal & $O(n m)$ & $O\left(n^{3}\right)$ \\
\hline power-4-chordal & $P$ & $O\left(n^{3}\right)$ \\
\hline power-weakly-chordal & $O\left(n^{3}\right)$ & $O\left(n^{3}\right)$ \\
\hline distance-hereditary & $O\left(n^{2}\right)$ & $O\left(n^{3}\right)$ \\
\hline AT-free & ${ }^{(*)} O\left(n^{3}\right)$ & $O\left(n^{2}\right)$ \\
\hline cocomparability & ${ }^{(*)} O\left(n^{3}\right)$ & \\
\hline interval & ${ }^{(*)} O\left(n^{2}\right)$ & $N P$ and $3 / 2$-approximation \\
\hline circular-arc & ${ }^{(*)}$ and $3 / 2$-approximation & . \\
\hline
\end{tabular}

For a given graph $G$ with $n$ vertices and $m$ edges, we first find the distance matrix of $G$, then construct the graphs $L(\mathcal{D}(G, r))$ and $\Gamma(\mathcal{D}(G, r))$ using that matrix in $O\left(n^{2}\right)$ time, and finally color $L(\mathcal{D}(G, r))$ and/or $\Gamma(\mathcal{D}(G, r))$ using some known algorithm (depending on what graph family the graph $G$ is from). Note that graphs $L(\mathcal{D}(G, r))$ and $\Gamma(\mathcal{D}(G, r))$ may have now $O\left(n^{2}\right)$ edges. To compute the distance matrix, for distance-hereditary graphs and interval graphs we can use $O\left(n^{2}\right)$ time algorithms presented in [17, for other graph families we use general $O(n m)$ time algorithm. To color chordal graphs (as well as interval 
graphs), we can use a linear (in the size of the constructed graph) time algorithm from [23]. To color weakly chordal graphs, we use an algorithm from 26. which will color $L(\mathcal{D}(G, r))$ and/or $\Gamma(\mathcal{D}(G, r))$ in $O\left(n^{3}\right)$ time. To color 4-chordal graphs, we can use general polynomial time coloring algorithm designed in 25] for all perfect graphs (4-chordal graphs are perfect). To color cocomparability graphs, we can use $O\left(n^{3}\right)$ time algorithm from [30. According to [1, it is computationally hard to approximately color the even powers of $n$-vertex chordal graphs within an $n^{\frac{1}{2}-\epsilon}$ factor, for any $\epsilon>0$. Consequently, it is computationally hard to approximately $r$-color any chordal (hence, any weakly chordal, any 4chordal) graph within the same $n^{\frac{1}{2}-\epsilon}$ factor. According to [6], to (approximately) color a dually chordal graph is as hard as to (approximately) color any graph. Since dually chordal graphs are square-chordal, it is computationally hard to approximately $r^{+}$-color any square-chordal (hence, any square-weakly-chordal, any square-4-chordal) graph. We know 22] that coloring of circular-arc graphs is an NP-complete problem. Since $\Gamma(\mathcal{D}(G, r)) \simeq G$ when $r(v)=0$ for every $v \in V$, the general $r^{+}$-Coloring problem is also NP-complete on circular-arc graphs. However, we do not know the complexity of the $r$-Coloring problem on circular-arc graphs $G$ as the graphs $L(\mathcal{D}(G, r))$ may represent only a subclass of circular-arc graphs. One can use the circular-arc graph coloring approximation algorithm of [27] with a performance ratio of $3 / 2$ to get an approximate solution for the corresponding $r$-Coloring and/or $r^{+}$-Coloring problems on circular-arc graphs with the same performance ratio.

In the full version of this paper, other applications of the generalized powers of graphs (e.g., to $r$-packing, $q$-dispersion, $k$-domination, $p$-centers, $r$-clustering, etc.) are discussed.

\section{References}

1. G. Agnarsson, R. Greenlaw and M.M. Halldorsson, On powers of chordal graphs and their colorings, Congressus Numerantium, 144 (2000), 41-65.

2. G. Agnarsson and M.M. Halldorsson, Coloring powers of planar graphs, SIAM J. Disc. Math. 16 (2003), 651-662.

3. H.J. Bandelt, A. Henkmann and F. Nicolai, Powers of distance-hereditary graphs, Discrete Math. 145 (1995), 37-60.

4. A.A. Bertossi, M.C. Pinotti and R.B. Tan, Channel Assignment with Separation for Interference Avoidance in Wireless Networks, IEEE Trans. Parallel Distrib. Syst. 14 (2003), 222-235.

5. H.L. Bodlaender, T. Kloks, R.B. Tan J.van Leeuwen, $\lambda$-Coloring of Graphs, STACS 2000, LNCS 1770, 395-406.

6. A. Brandstädt, V. Chepoi and F. Dragan, The algorithmic use of hypertree structure and maximum neighborhood orderings, Disc. Appl. Math. 82 (1998), 43-77.

7. A. Brandstädt, F.F. Dragan, V. Chepoi and V.I. Voloshin, Dually Chordal Graphs, SIAM J. Discrete Math. 11 (1998), 437-455.

8. A. Brandstädt, F.F. Dragan and F. Nicolai, Homogeneously orderable graphs, Theoretical Computer Science 172 (1997), 209-232.

9. A. Brandstädt, V.B. Le and J. Spinrad, Graph Classes: A Survey, SIAM Monographs on Discrete Math. Appl., (SIAM, Philadelphia, 1999). 
10. T. Calamoneri and R. Petreschi, On the Radiocoloring Problem, IWDC 2002, LNCS 2571, 118-127.

11. G.J. Chang and D. Kuo, The $L(2,1)$-labeling problem on graphs, SIAM J. Disc. Math. 9 (1996), 309-316.

12. J.M. Chang, C.W. Ho and M.T. Ko, Powers of Asteroidal Triple-free Graphs with Applications, Ars Combinatoria 67 (2003), 161-173.

13. J.M. Chang, C.W. Ho and M.T. Ko, LexBFS-ordering in Asteroidal Triple-Free Graphs, ISAAC 1999, LNCS 1741, 163-172.

14. M. Chen and G.J. Chang, Families of Graphs Closed Under Taking Powers, Graphs and Combinatorics 17 (2001), 207-212.

15. E. Dahlhaus, P. Duchet, On strongly chordal graphs, Ars Comb. 24B (1987), 23-30.

16. P. Damaschke, Distances in cocomparability graphs and their powers, Discrete Applied Mathematics 35 (1992), 67-72.

17. F.F. Dragan, Estimating All Pairs Shortest Paths in Restricted Graph Families: A Unified Approach, Journal of Algorithms 57 (2005), 1-21.

18. P. Duchet, Classical perfect graphs, Ann. Discrete Math. 21 (1984), 67-96.

19. J. Fiala, T. Kloks and J. Kratochvíl, Fixed-parameter complexity of $\lambda$-labelings, WG 1999, LNCS 1665, 350-363.

20. C. Flotow, On Powers of Circular Arc Graphs and Proper Circular Arc Graphs, Discrete Applied Mathematics 69 (1996), 199-207.

21. D. Fotakis, S.E. Nikoletseas, V.G. Papadopoulou and P.G. Spirakis, NP-Completeness Results and Efficient Approximations for Radiocoloring in Planar Graphs, MFCS 2000, LNCS 1893, 363-372.

22. M.R. Garey, D.S. Johnson, G.L. Miller and C.H. Papadimitriou, The complexity of coloring circular arcs and chords, SIAM J. Alg. Disc. Meth. 1 (1980), 216-227.

23. F. Gavril, Algorithms for min. coloring, max. clique, min. covering by cliques and max. independent set of a chordal graph, SIAM J. Comput. 1 (1972), 180-187.

24. J.R. Griggs and R.K. Yeh, Labeling graphs with a condition at distance 2, SIAM J. Discrete Math. 5 (1992), 586-595.

25. M. Grötschel, L. Lovasz and A. Schrijver, Polynomial algorithms for perfect graphs, In Topics on perfect graphs, volume 88, pages 325-356.

26. R. Hayward, J. Spinrad R. Sritharan, Weakly chordal graph algorithms via handles, SODA 2000, 42-49.

27. I.A. Karapetian, On Coloring of Arc Graphs, Akademiia nauk Armianskoi SSR Doklady, 70 (1980), 306-311.

28. D. Král', Coloring Powers of Chordal Graphs, SIAM Journal on Discrete Mathematics, 18 (2004), 451-461.

29. R. Laskar and D. Shier, Powers and centers of chordal graphs, Discrete Appl. Math. 6 (1983), 139-147.

30. R.H. Möhring, Algorithmic aspects of comparability graphs and interval graphs, Graphs and Order, (I. Rival, ed.,) (1985) 41-102.

31. S. Ramanathan and E.L. Lloyd, Scheduling Algorithms for Multihop Radio Networks, IEEE/ACM Transactions on Networking, 1 (1993), 166-172.

32. A. Raychaudhuri, On powers of strongly chordal and circular arc graphs, Ars Combin. 34 (1992), 147-160.

33. I. Todinca, Coloring Powers of Graphs of Bounded Clique-Width, WG 2003, LNCS $2880,370-382$. 